\title{
Aarroo Perfil de encaminhamentos a fisioterapia por um serviço de Atenção Primária à Saúde, 2012*
}

\section{Profile of patients referred to physiotherapy by a Primary Health Care Service in 2012}

Guilherme Grivicich da Silva

Grupo Hospitalar Conceição, Hospital Cristo Redentor, Porto Alegre-RS, Brasil

Sergio Antonio Sirena

Grupo Hospitalar Conceição, Gerência de Ensino e Pesquisa, Porto Alegre-RS, Brasil

\section{Resumo}

Objetivo: descrever a frequência de encaminhamentos e as características dos usuários da Atenção Primária à Saúde (APS) encaminhados aos serviços de fisioterapia, por Unidades de Saúde do Serviço de Saúde Comunitária do Grupo Hospitalar Conceição em Porto Alegre-RS, Brasil. Métodos: estudo transversal descritivo com dados primários e secundários, obtidos a partir de questionários e dos formulários de encaminhamento referentes ao período de julho a setembro de 2012. Resultados: do total de 258 formulários, a frequência de encaminhamentos a fisioterapia foi de 1,1\%; predominaram mulheres (70,5\%) não idosas $(59,4 \%)$; os diagnósticos clínicos mais prevalentes foram a osteoartrose $(29,1 \%)$ e as tendinopatias/lesões de tecidos moles $(27,1 \%) ; 87,1 \%$ dos usuários referiram dor, estando 48,1\% destes, pelo menos, parcialmente impossibilitados de trabalhar. Conclusão: a frequência de encaminhamentos à fisioterapia pela APS foi baixa, embora a população esteja em um processo de adoecimento por problemas crônicos, com presença de dor e redução da capacidade laboral.

Palavras-chave: Epidemiologia Descritiva; Necessidades e Demandas de Serviços de Saúde; Fisioterapia; Atenção Primária à Saúde.

\begin{abstract}
Objective: to describe the frequency of referrals and the characteristics of patients referred to physiotherapy services by Conceição Hospital Group Community Health Service Primary Health Care Units (PHCU). Methods: this is a cross-sectional and descriptive study using primary and secondary data obtained from questionnaires and referral forms for the period July to September 2012. Results: analyzing 258 forms, we found 1.1\% frequency of referral to physical therapy with higher prevalence of women (70.5\%) and non-elderly women (59.4\%). The two most prevalent clinical diagnoses were osteoarthrosis (29.1\%) and tendinosis/soft tissue injuries (27.1\%). 87.1\% of patients referred pain and at last $48.1 \%$ of these were partially unable to work. Conclusion: prevalence of PHCU referrals to physiotherapy is low, although the population is subject to chronic illness problems, with pain as an aggravating factor and interference in work capacity.
\end{abstract}

Key words: Epidemiology, Descriptive; Health Service Needs and Demands; Physical Therapy Specialty; Primary Health Care.

\footnotetext{
* Manuscrito proveniente da dissertação de Mestrado Profissional em Epidemiologia pela Universidade Federal do Rio Grande do Sul (UFRGS), com ênfase em Gestão de Tecnologia de Saúde na Atenção Primária à Saúde, em parceria com o Grupo Hospitalar Conceição (GHC) e com o Hospital de Clínicas de Porto Alegre (HCPA), apresentada por Guilherme Grivicich da Silva, em 2013.
}

Endereço para correspondência:

Guilherme Grivicich da Silva - Grupo Hospitalar Conceição, Hospital Cristo Redentor, Fisioterapia e Reabilitação, Rua Domingos Rubbo, $n^{\circ}$ 20, Cristo Redentor, Porto Alegre-RS, Brasil. CEP:91040-000

E-mail:sguilherme@ghc.com.br 


\section{Introdução}

A prevalência de utilização dos serviços de fisioterapia na população brasileira geral é baixa quando comparada à de algumas cidades da região Sul do Brasil. Em estudo para avaliar os custos com fisioterapia pelo Sistema Único de Saúde (SUS) em 2008, verificou-se que aproximadamente 1 em cada 5 usuários utilizava esse serviço (19\%). ${ }^{1}$ Essa frequência de utilização variou entre 27,3\% em Guarapuava-PR, ${ }^{2}$ 30,2\% em Pelotas-RS ${ }^{3}$ e 33,5\% em Lajes-SC. ${ }^{4}$ Este resultado é relevante, visto que a fisioterapia pode desempenhar um papel importante na promoção, prevenção, reabilitação e melhoria da qualidade de vida dos usuários, reduzindo o tempo de internação hospitalar e os custos do cuidado.

No Brasil, a oferta de atendimento fisioterápico ambulatorial pelo SUS é pequena todavia, e geograficamente desigual. 0 sistema remunera inadequadamente os serviços prestados em fisioterapia e ainda o faz, em grande parte, por meio de convênios com estabelecimentos privados. ${ }^{1}$ Um estudo brasileiro de base populacional identificou que a prevalência de utilização dos serviços de fisioterapia ao longo da vida foi de $30,2 \%$, sendo que $4,9 \%$ dos entrevistados usaram algum serviço de fisioterapia nos 12 meses anteriores à entrevista. ${ }^{3}$ Ainda assim, a literatura carece de mais pesquisas para identificar a frequência de utilização desses serviços.

\section{Desde 2008, o fisioterapeuta pode compor o Núcleo de Apoio à Saúde da Familia (NASF).}

Desde a publicação da Portaria do Ministério da Saúde SAS/MS n ${ }^{0}$ 154, de 24 de janeiro de 2008, o fisioterapeuta pode compor o Núcleo de Apoio à Saúde da Família (NASF), de acordo com a demanda e organização da rede integrada de saúde, realizando função de matriciamento. ${ }^{5}$ Embora na teoria, a participação da fisioterapia na Atenção Primária à Saúde (APS) esteja fundamentada, na prática, a profissão consolida seus primeiros passos na conquista de seu espaço nesse nível de atenção à saúde.

Remonta ao surgimento da profissão o papel do fisioterapeuta vinculado à imagem de profissional reabilitador, responsável por tratar problemas, incapacidades e deficiências já instaladas. Cabe à fisioterapia a revisão de seus fundamentos e uma análise aprofundada de sua prática profissional, visando adaptações à nova realidade, de forma a contribuir para as mudanças no quadro social e sanitário do país. Essa readaptação deve basear-se na competência e responsabilidade do fisioterapeuta. A responsabilização estimula novas perspectivas de relacionamento entre profissionais e usuários, estabelece novos vínculos, e estimula o surgimento de ações promocionais e preventivas. ${ }^{7.8}$

0 presente estudo tem por objetivo descrever a frequência de encaminhamentos e as características dos usuários da Atenção Primária à Saúde - APS encaminhados a serviços de fisioterapia, por Unidades de Saúde (US) do Serviço de Saúde Comunitária do Grupo Hospitalar Conceição (SSC/GHC).

\section{Métodos}

Trata-se de um estudo transversal e descritivo, com base em dados secundários, provenientes do formulário de encaminhamento; e em dados primários, coletados pelos médicos e residentes das US participantes, a partir das respostas a questionário específico elaborado para caracterização do problema apresentado pelos usuários encaminhados à fisioterapia.

A pesquisa foi realizada nas US do Serviço de Saúde Comunitária do Grupo Hospitalar Conceição - SSC/ GHC -, localizado na cidade de Porto Alegre, estado do Rio Grande do Sul, Brasil, no período de julho a setembro de 2012. $0 \mathrm{SSC} / \mathrm{GHC}$ é formado por 12 US, todas compostas por equipes básicas-padrão, sugeridas pelo Ministério da Saúde, constituídas por médicos com especialização em medicina de família e comunidade e programa de residência médica de referência no âmbito nacional. Todas as US localizam-se na zona norte de Porto Alegre e prestam atendimento à população pelo SUS. Trata-se de um serviço de saúde comunitária bem estruturado e organizado, com maior grau de orientação à APS na comparação com os demais serviços desse nível de atenção disponíveis no município. ${ }^{9}$

Os pesquisadores visitaram as 12 US para apresentar a pesquisa e dirimir possíveis dúvidas relativas ao preenchimento dos formulários. As equipes eram consultadas quanto à viabilidade de desenvolver a coleta de dados, de maneira que 8 das 12 Unidades 
de Saúde do SSC/GHC aceitaram participar da pesquisa. As outras quatro US não participaram do estudo por alegar sobrecarga rotineira de trabalho. As 8 US participantes atendiam a uma população cadastrada total de 54.410 usuários, com predomínio de mulheres (54,5\%) e indivíduos não idosos (81,4\%), e média de idade de 54,8 anos com desvio-padrão (dp) de 15,8 anos (dados do Sistema de Informações do SSC/GHC).

Durante o período do estudo, somente os formulários de coleta eram disponibilizados nessas unidades participantes. Portanto, todos os encaminhamentos à fisioterapia dessas unidades fizeram parte da presente pesquisa.

0 formulário de coleta consistia no formulário de encaminhamento-padrão (fornecido pela Secretaria Municipal de Saúde), grampeado a uma folha de carbono, e a um formulário de pesquisa (formulário de encaminhamento-padrão na frente e questionário no verso); e dois Termos de Consentimento Livre e Esclarecido (TCLE).

Os dados contidos no formulário de encaminhamento-padrão, para fins desta pesquisa, foram: sexo; idade; diagnóstico clínico; história clínica; objetivo da solicitação de fisioterapia; número de sessões solicitadas; e número de sessões já realizadas pelo mesmo motivo. Todas essas informações contavam com campo aberto para escrita. A categorização foi feita posteriormente, para a análise dos dados. Esse formulário era preenchido exclusivamente pelo médico ou residente.

0 questionário, por sua vez, era composto de 8 campos de respostas rápidas e objetivas em relação à dor e repercussões no trabalho. Entre as variáveis a serem preenchidas, era necessário escrever a principal ocupação e assinalar o tipo de fisioterapia solicitada (respiratória ou motora). Em relação às perguntas, todas se referiam ao problema pelo qual o usuário era referenciado à fisioterapia: 'Já consultou pelo mesmo motivo?' (Sim ou Não); número de consultas médicas pelo mesmo motivo (livre escrita, sem categorias pré-determinadas); 'Presença de dor?' (Sim ou Não); 'Quanto de dor já sentiu decorrente desse problema?' (nenhuma; muito leve; leve; moderada; grave; ou muito grave); 'Quanto a dor interferiu com seu trabalho normal, incluindo o trabalho de dentro de casa?' (de maneira alguma; um pouco; moderadamente; bastante; extremamente); 'Está impossibilitado de trabalhar?' (Sim totalmente; Sim parcialmente; ou Não).
Como as respostas a essas perguntas dependiam da colaboração do usuário (dados primários), essa etapa de preenchimento era realizada pelo médico e ou residente com o auxílio do usuário, familiar ou responsável. Após serem informados da pesquisa, os usuários assinavam o TCLE. 0 TCLE era apresentado em duas vias, sendo uma cópia entregue ao sujeito de pesquisa ou seu responsável (se fosse 0 caso) após sua assinatura.

0 formulário de encaminhamento-padrão era preenchido pelo médico sempre que, como resultado da consulta médica em atenção básica, o usuário fosse referenciado à fisioterapia. Ao preencher esse formulário, em virtude da folha de carbono anexada, uma segunda via desse documento era automaticamente gerada. Além disso, o usuário era questionado sobre a possibilidade de participar da pesquisa e respondia a perguntas referentes ao motivo pelo qual era referenciado à fisioterapia. 0 médico (contratado ou residente) que solicitasse o referenciamento se responsabilizava pelo preenchimento do questionário, quando o usuário aceitasse participar da pesquisa. Com a negativa do usuário, apenas a segunda via do formulário de encaminhamento-padrão era utilizada, no sentido de contabilizar o número total de encaminhados, verificar o diagnóstico clínico e variáveis demográficas. Das 8 US participantes, em 3 delas foi preenchido somente 0 registro do formulário de encaminhamento-padrão à fisioterapia, alegando inviabilidade da aplicação do questionário por implicar aumento no tempo de consulta, o que prejudicaria a rotina de trabalho da US. As demais unidades preencheram o formulário-padrão e o questionário.

Para cálculo da frequência de encaminhamentos à fisioterapia, foi utilizado o número total de consultas médicas na atenção básica, nas 8 unidades participantes, no período de julho a setembro de 2012, obtido do Sistema de Informações do GHC. A frequência de encaminhamentos foi obtida pela divisão do número total de encaminhamentos nessas 8 unidades participantes, no período da coleta, pelo número total de consultas médicas realizadas no período estudado.

A análise dos dados, realizada pelo programa Statistical Package for Social Sciences (SPSS) versão 18.0, consistiu em estatística descritiva com frequências e intervalo de confiança de $95 \%$ ( $\mathrm{IC}_{95 \%}$ ), médias e desvio-padrão da média e percentuais, para avaliar a frequência de encaminhamentos e as variá- 
veis demográficas da demanda. Para as associações brutas das variáveis categóricas por sexo, faixa etária e diagnósticos clínicos mais prevalentes, utilizou-se o teste do qui-quadrado. E para testar a normalidade das variáveis contínuas, o teste de Shapiro-Wilk. As variáveis contínuas sem distribuição normal foram comparadas pelo teste de Mann-Whitney. Em todos os testes estatísticos, considerou-se um nível de significância de 5\%.

0 projeto do estudo foi aprovado pelo Comitê de Ética em Pesquisa do Grupo Hospitalar Conceição, sob parecer consubstanciado de $\mathrm{n}^{\circ}$ 25730/2012, de acordo com a Resolução do Conselho Nacional de Saúde (CNS) n ${ }^{\circ}$ 466, de 12 de dezembro de 2012, que estabelece normas e conceitos éticos para pesquisas com seres humanos.

\section{Resultados}

Em 5 das 8 Unidades de Saúde participantes, a dinâmica de trabalho e o fluxo de pacientes permitiram o preenchimento do questionário, viabilizando a obtenção de outros dados (provenientes das respostas ao questionário) capazes de caracterizar os usuários quanto ao problema pelo qual foram referenciados à fisioterapia. Nessas 5 US, não houve, por parte dos usuários, qualquer recusa em participar do trabalho, totalizando 76 usuários que aceitaram participar e responderam ao instrumento de pesquisa.

A Tabela 1 demonstra a frequência de encaminhamentos, considerando as US participantes e 0 total de encaminhamentos. 0 percentual de usuários encaminhados para fisioterapia foi de $1,1 \%(n=258)$ (Tabela 1).

Os dados referentes às variáveis do formulário de encaminhamento-padrão dos 258 usuários encaminhados para fisioterapia são apresentados na Tabela 2, com predominância das seguintes características: sexo feminino $(70,5 \%)$, menores de 60 anos de idade $(59,4 \%)$, com registro de dor em sua história clínica $(78,7 \%)$, sendo a osteoartrose $(29,1 \%)$ e as tendinopatias/lesões de tecidos moles $(27,1 \%)$ as doenças de maior prevalência.

A partir dos dados apresentados na Tabela 3, evidenciou-se o perfil dos 76 usuários. A dor estava presente em 67 , sendo de moderada a muito grave em 65, e interferiu no trabalho de 55 usuários ao menos moderadamente; 37 demonstraram-se ao menos parcialmente impossibilitados de exercer suas atividades laborais.

Nas comparações dos diagnósticos clínicos por faixa etária, os idosos apresentaram prevalência estatisticamente superior de osteoartrose (40/105), quando comparados aos indivíduos não idosos (35/153) $(\mathrm{p}=0,054)$. Por sua vez, os usuários com idade inferior a 60 anos apresentaram maior prevalência de lombalgia (29/153) quando comparados aos indivíduos idosos $(10 / 105)(\mathrm{p}=0,041)$. Nessa comparação por sexo, as mulheres (10/182) apresentaram uma frequência de cervicalgia estatisticamente superior à correspondente para os homens, nos quais não se verificou a ocorrência da doença $(p=0,040)$.

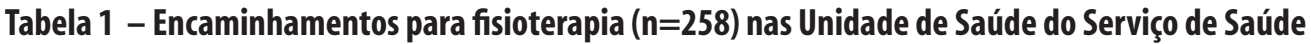 Comunitária/Grupo Hospitalar Conceição no município de Porto Alegre, Rio Grande do Sul, julho a setembro de 2012}

\begin{tabular}{lccccc}
\hline Unidade de Saúde & $\begin{array}{c}\text { População cadastrada } \\
(\mathbf{n})\end{array}$ & $\begin{array}{c}\text { Consultas } \\
(\mathbf{n})\end{array}$ & $\begin{array}{c}\text { Encaminhamentos } \\
(\mathbf{n})\end{array}$ & $\begin{array}{c}\text { Frequência } \\
(\%)\end{array}$ & $\begin{array}{c}\text { Encaminhamentos/ } \\
\text { Consultas } \\
(\text { por 1000) }\end{array}$ \\
\hline Barão de Bagé & 4.000 & 2.750 & 24 & 0,8 & 8,7 \\
Coinma & 5.778 & 3.545 & 14 & 0,3 & 3,9 \\
Floresta & 7.119 & 3.445 & 18 & 0,5 & 5,2 \\
Jardim Itu & 8.237 & 2.492 & 48 & 1,9 & 19,2 \\
Jardim Leopoldina & 13.157 & 3.472 & 96 & 2,7 & 27,6 \\
Parque dos Maias & 6.785 & 4.006 & 18 & 0,4 & 4,4 \\
Santíssima Trindade & 3.863 & 1.728 & 6 & 0,3 & 3,4 \\
SESC & 5.471 & 1.657 & 34 & 2,0 & 20,5 \\
\hline Total & 54.410 & 23.095 & 258 & 1,1 & 11,1 \\
\hline
\end{tabular}


Tabela 2 - Perfil dos encaminhamentos para fisioterapia $(n=258)$ no Serviço de Saúde Comunitária/Grupo Hospitalar Conceição no município de Porto Alegre, Rio Grande do Sul, julho a setembro de 2012

\begin{tabular}{|c|c|c|}
\hline Variável & $\mathbf{n}$ & $\%$ \\
\hline \multicolumn{3}{|l|}{ Sexo } \\
\hline Feminino & 182 & 70,5 \\
\hline Masculino & 76 & 29,5 \\
\hline \multicolumn{3}{|l|}{ Idade (em anos) } \\
\hline$<60$ & 153 & 59,4 \\
\hline$\geq 60$ & 105 & 40,6 \\
\hline \multicolumn{3}{|l|}{ História clínica } \\
\hline Dor & 152 & 58,9 \\
\hline Dor + outra queixa & 35 & 13,6 \\
\hline Lesão neurológica & 27 & 10,5 \\
\hline Dor + limitação funcional & 16 & 6,2 \\
\hline Evento traumático & 12 & 4,6 \\
\hline Outra & 16 & 6,2 \\
\hline \multicolumn{3}{|l|}{ Diagnóstico clínico } \\
\hline Osteoartrose & 75 & 29,0 \\
\hline Tendinopatias/Lesões de tecidos moles & 70 & 27,1 \\
\hline Lombalgia & 39 & 15,1 \\
\hline Doença neurológica & 20 & 7,8 \\
\hline Cervicalgia & 19 & 7,4 \\
\hline Dor crônica & 9 & 3,5 \\
\hline Fraturas & 8 & 3,1 \\
\hline Outra & 18 & 7,0 \\
\hline \multicolumn{3}{|l|}{ Objetivos da solicitação de fisioterapia } \\
\hline Analgesia + Recuperação funcional & 94 & 36,4 \\
\hline Analgesia & 83 & 32,2 \\
\hline Analgesia + Outro objetivo & 22 & 8,5 \\
\hline Reabilitação & 12 & 4,7 \\
\hline Outros & 47 & 18,2 \\
\hline \multicolumn{3}{|l|}{ Número de sessões solicitadas } \\
\hline Até 10 & 16 & 6,2 \\
\hline De 11 a 20 & 238 & 92,2 \\
\hline De 21 a 30 & 4 & 1,6 \\
\hline \multicolumn{3}{|l|}{ Número de sessões prévias } \\
\hline Nenhuma & 171 & 66,3 \\
\hline Até 10 & 14 & 5,4 \\
\hline De 11 a 20 & 54 & 21,0 \\
\hline Mais de 20 & 19 & 7,3 \\
\hline
\end{tabular}

A Tabela 4 demonstra as variáveis categóricas da amostra, comparadas por sexo e faixa etária. Em relação à impossibilidade de trabalhar, 9 homens estavam pelo menos parcialmente impossibilitados, frente a 4 mulheres $(\mathrm{p}=0,022)$. 0 número de idosos que estavam impossibilitados de trabalhar (12/20) foi superior ao dos mais jovens $(12 / 40)(\mathrm{p}=0,052)$ (Tabela 4$)$.

Não foram observadas diferenças estatisticamente significativas $(\mathrm{p}>0,05)$ entre as variáveis 'consultas pelo mesmo motivo', 'tempo de início da dor' e 'tempo impossibilitado de trabalhar' em relação ao sexo, faixa etária e os dois diagnósticos clínicos de maior prevalência (Tabela 5).

\section{Discussão}

0 presente estudo revelou frequência de encaminhamentos para fisioterapia de $1,1 \%$, com grande 
Tabela 3 - Características dos encaminhamentos para fisioterapia $(n=76)$ em relação a dor e repercussões no trabalho, no Serviço de Saúde Comunitária/Grupo Hospitalar Conceição no município de Porto Alegre, Rio Grande do Sul, julho a setembro de 2012

\begin{tabular}{|c|c|}
\hline Variável do questionário & $\mathbf{n}$ \\
\hline \multicolumn{2}{|l|}{ Ocupação } \\
\hline Nenhuma & 13 \\
\hline Aposentado(a) & 25 \\
\hline Doméstica/Faxineira/Serviços Gerais & 13 \\
\hline Outra & 25 \\
\hline \multicolumn{2}{|l|}{ Tipo de fisioterapia } \\
\hline Motora & 73 \\
\hline Respiratória & 3 \\
\hline \multicolumn{2}{|l|}{ Já consultou pelo mesmo motivo? } \\
\hline Sim & 67 \\
\hline Não & 9 \\
\hline \multicolumn{2}{|c|}{ Número de consultas médicas pelo mesmo motivo } \\
\hline Nenhuma & 9 \\
\hline Até 5 & 47 \\
\hline De 6 a 10 & 14 \\
\hline Mais de 10 & 6 \\
\hline \multicolumn{2}{|l|}{ Presença de dor? } \\
\hline Sim & 67 \\
\hline Não & 9 \\
\hline \multicolumn{2}{|c|}{ Quanto de dor já sentiu decorrente desse problema? } \\
\hline Não responderam & 8 \\
\hline Nenhuma & - \\
\hline Muito leve & 1 \\
\hline Leve & 2 \\
\hline Moderada & 26 \\
\hline Grave & 25 \\
\hline Muito grave & 14 \\
\hline \multicolumn{2}{|c|}{ Quanto a dor interferiu com seu trabalho normal, incluindo o trabalho dentro de casa? } \\
\hline Não responderam & 8 \\
\hline De maneira alguma & 6 \\
\hline Um pouco & 7 \\
\hline Moderadamente & 22 \\
\hline Bastante & 20 \\
\hline Extremamente & 13 \\
\hline \multicolumn{2}{|l|}{ Está impossibilitado de trabalhar? } \\
\hline Sim, totalmente & 13 \\
\hline Sim, parcialmente & 24 \\
\hline Não & 24 \\
\hline Não se aplica & 15 \\
\hline
\end{tabular}


Tabela 4 - Comparação de características dos encaminhamentos para fisioterapia ( $n=76)$, de acordo com sexo e idade (em anos), no Serviço de Saúde Comunitária/Grupo Hospitalar Conceição no município de Porto Alegre, Rio Grande do Sul, julho a setembro de 2012

\begin{tabular}{|c|c|c|c|c|c|c|}
\hline \multirow[b]{2}{*}{ Variável categórica do formulário de pesquisa } & \multicolumn{3}{|c|}{ Sexo } & \multicolumn{3}{|c|}{ Idade } \\
\hline & $\begin{array}{c}\text { Masculino } \\
\mathbf{n}\end{array}$ & $\underset{n}{\text { Feminino }}$ & $p^{a}$ & $\begin{array}{c}<60 \\
n\end{array}$ & $\begin{array}{c}\geq 60 \\
n\end{array}$ & $\mathbf{p a}$ \\
\hline \multicolumn{7}{|l|}{ Consultou pelo mesmo motivo? } \\
\hline Sim & 41 & 26 & \multirow{3}{*}{0,543} & 43 & 24 & \multirow{3}{*}{0,433} \\
\hline & & & & & & \\
\hline Não & 4 & 5 & & 4 & 5 & \\
\hline \multicolumn{7}{|l|}{ Impossibilitado de trabalhar? } \\
\hline Sim, parcialmente & $9^{*}$ & 4 & \multirow{3}{*}{0,024} & 11 & 2 & \multirow{3}{*}{0,051} \\
\hline Sim, totalmente & 12 & 12 & & 18 & 6 & \\
\hline Não & 6 & 18 & & 12 & 12 & \\
\hline \multicolumn{7}{|l|}{ Já realizou fisioterapia pelo mesmo motivo? } \\
\hline Sim & 17 & 23 & \multirow{3}{*}{0,741} & 26 & 14 & \multirow{3}{*}{0,554} \\
\hline & & & & & & \\
\hline Não & 14 & 22 & & 21 & 36 & \\
\hline \multicolumn{7}{|l|}{ Já realizou fisioterapia por outro motivo? } \\
\hline Sim & 4 & 18 & \multirow{3}{*}{0,023} & 11 & 11 & \multirow{3}{*}{0,172} \\
\hline & & & & & & \\
\hline Não & $27^{*}$ & 27 & & 36 & 18 & \\
\hline
\end{tabular}

a) Teste do qui-quadrado de Pearson

Tabela 5 - Comparação entre os diagnósticos clínicos ( $n=76$ ), de acordo com sexo (masculino ou feminino) e idade (em anos), no Serviço de Saúde Comunitária/Grupo Hospitalar Conceição no município de Porto Alegre, Rio Grande do Sul, julho a setembro de 2012

\begin{tabular}{|c|c|c|c|c|c|c|c|c|c|}
\hline Variável & $\begin{array}{c}\text { Osteoartrose } \\
M^{\text {b }} \\
\left(P_{25} ; P_{75}\right)\end{array}$ & $\begin{array}{c}\text { Tendinopatias/ } \\
\text { Lesões de } \\
\text { tecidos moles } \\
M^{\mathrm{b}} \\
\left(\mathrm{P}_{25} ; \mathrm{P}_{75}\right)\end{array}$ & $\mathrm{pa}$ & $\begin{array}{c}\text { Masculino } \\
\text { Md }^{\mathrm{b}} \\
\left(\mathbf{P}_{25} ; \mathbf{P}_{75}\right)\end{array}$ & $\begin{array}{c}\text { Feminino } \\
M d^{b} \\
\left(P_{25} ; P_{75}\right)\end{array}$ & $\mathbf{p a}$ & $\begin{array}{c}<60 \text { anos } \\
M d^{b} \\
\left(P_{25} ; P_{75}\right)\end{array}$ & $\begin{array}{c}\geq 60 \text { anos } \\
M d^{b} \\
\left(P_{25} ; P_{75}\right)\end{array}$ & pa \\
\hline $\begin{array}{l}\text { Consultas pelo mesmo } \\
\text { motivo }\end{array}$ & $\begin{array}{c}4 \\
(3,0 ; 5,0)\end{array}$ & $\begin{array}{c}3 \\
(2,3 ; 5,8)\end{array}$ & 0,369 & $\begin{array}{c}3 \\
(3 ; 6,2)\end{array}$ & $\begin{array}{c}5 \\
(3,0 ; 7,0)\end{array}$ & 0,564 & $\begin{array}{c}4 \\
(3,0 ; 9,0)\end{array}$ & $\begin{array}{c}4,5 \\
(2,5 ; 5,0)\end{array}$ & 0,502 \\
\hline Tempo (em meses) da dor & $\begin{array}{c}24 \\
(7,5 ; 57,0)\end{array}$ & $\begin{array}{c}6.5 \\
(2,3 ; 36,0)\end{array}$ & 0,073 & $\begin{array}{c}9 \\
(4 ; 36)\end{array}$ & $\begin{array}{c}24 \\
(5,0 ; 66,0)\end{array}$ & 0,151 & $\begin{array}{c}18 \\
(6,0 ; 36,0)\end{array}$ & $\begin{array}{c}12 \\
(2,5 ; 114,0)\end{array}$ & 0,938 \\
\hline $\begin{array}{l}\text { Tempo (em meses) } \\
\text { impossibilitado de } \\
\text { trabalhar }\end{array}$ & $\begin{array}{c}4 \\
(0,4 ; 34,5)\end{array}$ & $\begin{array}{c}2.5 \\
(0,5 ; 8,5)\end{array}$ & 1,002 & $\begin{array}{c}5 \\
(0,6 ; 11,3)\end{array}$ & $\begin{array}{c}5 \\
(1,5 ; 18,0)\end{array}$ & 0,681 & $\begin{array}{c}5,5 \\
(2,0 ; 12,0)\end{array}$ & $\begin{array}{c}3 \\
(0,5 ; 6,5)\end{array}$ & 0,323 \\
\hline
\end{tabular}

a) P-valor: teste de Mann-Whitney

b) Mediana 
variabilidade entre as US participantes, sendo a osteoartrose e as tendinopatias ou lesões de tecidos moles os diagnósticos clínicos de maior prevalência. A dor foi o sintoma presente em mais de dois terços dos usuários referenciados, e quase metade encontrava-se pelo menos parcialmente impossibilitada de trabalhar.

Por se tratar de um serviço de saúde bem estruturado, homogêneo e com forte grau de orientação à APS, ${ }^{9}$ esperava-se que tal processo de referenciamento pudesse ser de pouca variabilidade entre as US participantes. Estudo de base populacional realizado em Pelotas-RS encontrou prevalência de 30,2\% de necessidade de utilização dos serviços de fisioterapia ao longo da vida, e de 4,9\% de utilização desses serviços nos 12 meses que antecederam a entrevista deste estudo. ${ }^{3}$ A frequência de encaminhamentos encontrada e apresentada aqui é relativamente baixa. Talvez a falta de credibilidade e confiança nas clínicas que prestam serviços de fisioterapia ao SUS sejam fatores que desestimulem sua indicação e encaminhamento por parte dos médicos da APS. Além disso, a dificuldade em saber o que efetivamente acontece com aquele usuário encaminhado, se ele tem acesso ao atendimento fisioterapêutico ou se este é resolutivo, pode desencorajar 0 referenciamento.

0 número expressivo de usuários com osteoartrose e tendinopatias/lesões de tecidos moles reflete a realidade da população brasileira em processo de envelhecimento, o que favorece 0 aparecimento de doenças crônicas não transmissíveis (DCNT). A sobrecarga de trabalho facilita o surgimento das tendinopatias e lesões de tecidos moles. A falta de informação e orientações adequadas à população também limita uma maior efetividade de ações de promoção e prevenção em saúde. A osteoartrose (29,2\%) e as lesões musculoesqueléticas $(28,3 \%)$ apresentaram-se como as doenças mais prevalentes segundo levantamento epidemiológico de doenças relacionadas à fisioterapia, em estudo realizado junto a uma unidade básica de saúde na cidade de Campinas-SP. ${ }^{10}$

Tanto a prevalência quanto o nível de incidência $\mathrm{da}$ artrose aumentam a partir dos 40 anos de idade. A prevalência dessa doença possui padrões específicos, relacionados tanto à idade quanto ao sexo: antes dos 50 anos, essa prevalência é maior em homens; após os 50 anos, são as mulheres as mais afetadas pela artrose, nas mãos, pés e joelhos. ${ }^{11}$ Tais achados assemelham-se aos resultados do presente estudo, que encontrou uma prevalência estatisticamente superior de osteoartrose em indivíduos com mais de 60 anos de idade $(38,1 \%)$; porém, não foram identificadas diferenças estatisticamente significativas em relação a osteoartrose na comparação entre sexos.

Houve um predomínio de encaminhamentos de mulheres (70,5\%), com média de idade de 54,8 anos. Estudo realizado em uma unidade básica de saúde de Sorocaba-SP, com 40 pacientes apresentando dor musculoesquelética, também observou predominância do sexo feminino $(82,2 \%)$, com média de idade de 54,8 anos ${ }^{12}$ Se por um lado, histórica e culturalmente responsáveis pelo cuidado, as mulheres mantém uma postura mais cautelosa em relação a seu corpo e tendem a identificar, preocupar-se e procurar atendimento em saúde com uma frequência maior que os homens, por outro lado, o estudo acerca da utilização de fisioterapia realizado em Lages-SC demonstra que os homens $(36,4 \%)$ utilizam mais a fisioterapia do que as mulheres (31,5\%).4 Essas peculiaridades de gênero podem ter influência nos achados deste estudo.

Também ao considerar o levantamento epidemiológico realizado em Campinas-SP, a distribuição de agravos à saúde por faixa etária demonstra: maior prevalência de doenças osteomusculares na população de 20 a 39 anos (6,6\%) e de 40 a 59 anos (9,4\%); maior prevalência de artrose aos 60 anos ou mais $(21,7 \%)$; e maior prevalência de fraturas em mulheres idosas. ${ }^{10}$ Esses resultados são semelhantes aos do presente estudo, que revelou maior prevalência de osteoartrose em idosos $(38,1 \%)$ e problemas osteomusculares entre indivíduos não idosos (lombalgia, 19\%); não obstante, de acordo com o presente estudo, as fraturas mostraram-se mais prevalentes no sexo masculino, independentemente das faixas etárias.

As doenças osteomusculares tendem a aparecer na idade economicamente ativa, caracterizada por maior força produtiva e poder para o trabalho. Adultos jovens tendem a priorizar o crescimento profissional e econômico e, por vezes, negligenciam os cuidados com a própria saúde. Assim, fragilizam seu corpo e facilitam o aparecimento de doenças osteomusculares. 0 processo de envelhecimento natural também leva a um desgaste articular e desmineralização óssea, principalmente entre mulheres pós-menopáusicas, favorecendo a osteoartrose e maior incidência de fraturas.

Em relação à faixa etária, a maior frequência de encaminhamentos aconteceu para indivíduos não 
idosos (59,4\%), com acometimento importante por lombalgia. A dor lombar impõe uma grande carga social e econômica à sociedade e pode ser incapacitante. Diretrizes internacionais para o manejo da lombalgia destacam que essa condição deva ser manejada na Atenção Primária. Entretanto, alguns profissionais das unidades de saúde sentem necessidade de maiores informações sobre o diagnóstico e a efetividade dos tratamentos dessa condição. ${ }^{5}$

Os resultados obtidos com o presente estudo evidenciam a lombalgia como o terceiro diagnóstico de maior prevalência entre o total dos usuários encaminhados à fisioterapia $(15,1 \%)$. A maior parte dos problemas lombares são determinados por desequilíbrios musculares e pela execução - inadequada - de gestos laborais, hábitos posturais e atividades de lazer. Observa-se, claramente, a formação de uma frente de atuação do profissional fisioterapeuta na tentativa de mudar esse quadro de fatores. Ações de promoção e prevenção em saúde, como a implementação de escolas de postura e orientações sobre os cuidados necessários com a coluna, são de grande importância no sentido de mudar tais comprometimentos na população. Certamente, conhecimentos básicos sobre 0 funcionamento da coluna poderiam reduzir a incidência desses quadros de lombalgia, que sobrecarregam a assistência ambulatorial do sistema de saúde.

Os presentes achados demonstraram que, para quase dois terços dos usuários encaminhados à fisioterapia, a dor interferiu, pelo menos moderadamente, em seu trabalho normal (incluído o trabalho doméstico). Além disso, 48,1\% dos encaminhados ficaram impossibilitados, pelo menos parcialmente, de trabalhar em decorrência da dor. Na Austrália, um estudo de coorte, com acompanhamento de um ano, avaliou pacientes com dor lombar inespecífica que consultaram em unidades básicas de saúde. Seus autores, entre outros achados, verificaram que (i) 79,5\% dos participantes trabalhavam antes do aparecimento da lombalgia e (ii) $37,8 \%$ relataram mudanças no status de trabalho causadas pela doença. ${ }^{13}$

Os achados da presente pesquisa evidenciam que a tendinopatia ou lesões de tecidos moles, normalmente diagnosticada em membros superiores, representam $27,1 \%$ do total de encaminhamentos para fisioterapia, sendo a segunda morbidade mais frequente. Os custos associados à queixa de dor no ombro são altos, em decorrência do elevado percentual de afastamento do trabalho por esse problema. Estudo norte-americano sobre usuários da APS com dor no ombro percebeu que homens adultos jovens (com idade inferior a 52 anos) apresentam lesões prévias associadas a dor no ombro, sendo que um quinto deles demonstrou lesões relacionadas ao trabalho. ${ }^{14}$

Evidenciou-se que os usuários encaminhados à fisioterapia apresentavam dor em quase $90 \%$ dos casos, de intensidade moderada a muito grave em $85,2 \%$. A interferência da dor no trabalho, de moderada a grave em mais de dois terços deles, incapacita-os para o desempenho das atividades laborais, pelo menos parcialmente, na quase metade dos usuários encaminhados. Tais achados constituem um problema de Saúde Pública devido aos custos associados e à necessidade de se encontrar estratégias, principalmente na APS, que aumentem a resolutividade dos serviços de saúde sobre essas condições e seus agravos. Os dados levam a crer que os usuários, quando foram referenciados à fisioterapia, apresentavam problemas sérios já instalados, não permitindo qualquer tipo de prevenção primária. A tarefa do fisioterapeuta, então, limita-se à atenção curativa, reabilitadora, até paliativa, senão desautorizando ações de promoção e prevenção em saúde.

O compromisso das equipes da Estratégia Saúde da Família (ESF) está em oferecer à população uma atenção integral à saúde, baseada em uma abordagem multidisciplinar, decorrente tanto da reorganização do sistema de saúde brasileiro quanto das alterações no perfil de morbidade da população. A missão dos profissionais da ESF aponta para a possibilidade de um grande avanço na definição e descrição mais qualificada das ações inerentes ao profissional fisioterapeuta na Atenção Primária à Saúde, adequada a sua formação e processo de trabalho, e às reais necessidades da comunidade. ${ }^{6}$ A oportunidade de inserção da fisioterapia na ESF é evidente, tanto por sua contribuição efetiva para a resolubilidade da estratégia como para a consolidação dos princípios norteadores do SUS. Essa inserção, contudo, deve-se subsidiar de informações sobre as demandas da população e a infraestrutura local, de forma a assegurar a eficácia das ações encarregadas ao profissional. $^{15}$

Cabe ao fisioterapeuta, junto à equipe de saúde e aos gestores locais, planejar e desenvolver estratégias que contemplem ações de reabilitação, imprescindíveis de serem desenvolvidas, como também ações de pro- 
moção da saúde e prevenção de doenças. ${ }^{16}$ No Reino Unido, os profissionais de reabilitação atuam na APS com Médicos de Família, indicando uma tendência de mudança dos serviços de reabilitação do setor secundário para o primário. No Canadá, os fisioterapeutas são referenciados pelos médicos da APS: estudo desenvolvido naquele país, com o objetivo de analisar qual desses modelos ofereceria maior acesso aos serviços de fisioterapia, verificou que, independentemente da estratégia a ser utilizada, tudo depende da alocação de recursos e dos stakeholders - os maiores interessados, quais sejam, os fisioterapeutas -, aos quais cabe tal tarefa. Contudo, os autores do estudo canadense são categóricos em afirmar que o acesso à fisioterapia é necessário ao manejo de condições crônicas. ${ }^{17}$

Entre as limitações do presente estudo, destaca-se o fato de 4 das 12 Unidades de Saúde do Serviço de Saúde Comunitária do Grupo Hospitalar Conceição SSC/GHC - não participarem da pesquisa, ademais da dificuldade em prolongar a coleta de dados. 0 tempo necessário para preenchimento dos formulários de coleta e a logística da pesquisa impossibilitaram 0 alcance de uma amostra mais variada e numericamente mais expressiva. São problemas que poderiam ser solucionados se houvesse uma padronização e redução da burocracia nos encaminhamentos de fisioterapia via sistema informatizado. Essa informatização do processo de referência dos pacientes teria facilitado a adesão das demais unidades de saúde a esta pesquisa.

A principal contribuição deste trabalho foi caracterizar a demanda de encaminhamentos para fisioterapia oriundos da Atenção Primária. Embora a frequência

\section{Referências}

1. Castro AP, Neves VR, Aciole GG. Diferenças regionais e custos dos procedimentos de fisioterapia no Sistema Único de Saúde do Brasil, 1995 a 2008. Rev Panam Salud Publica. 2011 nov;30(5):469-76.

2. Bim CR, Pelloso SM, Previdelli ITS. Inquérito domiciliar sobre uso da Fisioterapia por mulheres em Guarapuava-Paraná-Brasil. Cienc Saude Coletiva. 2011 set;16(9):3837-44.

3. Siqueira FV, Facchini LA, Hallal PC. Epidemiologia da utilização de fisioterapia em adultos e idosos. Rev Saude Publica. 2005 ago;39(4):663-8.

4. Moretto LC, Longo GZ, Boing AF, Arruda MP. Prevalência da utilização de serviços de fisioterapia de encaminhamentos tenha sido relativamente baixa, a caracterização dos usuários encaminhados mostrou mudanças importantes em seu estado de saúde, na qualidade de vida e no grau dos prejuízos sociais e financeiros decorrentes do problema pelo qual foram referenciados. Espera-se que os gestores da saúde tenham acesso e reúnam os subsídios necessários à elaboração de estratégias e planos de ação preparados para consolidar a participação da fisioterapia na Atenção Primária à Saúde, pensando não somente no tratamento dessas patologias mais prevalentes como também em sua prevenção e na promoção da saúde.

\section{Agradecimentos}

Aos funcionários do Serviço de Saúde Comunitária do Grupo Hospitalar Conceição - SSC/GHC -, pelo auxílio na coleta dos dados.

Ao corpo docente do Programa de Pós-Graduação em Epidemiologia da Universidade Federal do Rio Grande do Sul - UFRGS -, pela oportunidade do Mestrado Profissional.

\section{Contribuição dos autores}

Os autores, Silva GG e Sirena SA, participaram da concepção e delineamento do estudo, análise e interpretação dos dados, redação e revisão crítica relevante do conteúdo intelectual do manuscrito.

Ambos aprovaram sua versão final e declaram serem responsáveis por todos os aspectos do trabalho, garantindo sua precisão e integridade.

entre a população adulta urbana de Lages, Santa Catarina. Rev Bras Fisioter. 2009 mar-abr;13(2):130-5.

5. Brasil. Ministério da Saúde. Portaria $n^{0} 154$, de 24 de janeiro de 2008. Cria os Núcleos de Apoio à Saúde da Família - NASF. Diário Oficial da República Federativa do Brasil, Brasília (DF), 2008 jan 25. Seção 1:18.

6. Novais BKLO, Brito GEG. Percepções sobre o trabalho do fisioterapeuta na atenção primária. Rev APS. 2011 out-dez;14(4):424-34.

7. Santos FC, Aleluia IRS, Santos IN, Moura LGF, Carvalho MA. Participação do fisioterapeuta na equipe multiprofissional de auditoria em saúde. RAS. 2011 abr-jun;13(51):95-102. 
8. Teixeira CF, Paim JS, Vilasbôas AL. SUS, modelos assistenciais e vigilância da saúde. Inf Epidemiol SUS. 1998 abr-jun;7(2):7-28.

9. Castro RC, Knauth DR, Hauser L, Duncan BB. Quality assessment of primary care by health professionals: a comparison of different types of services. Cad Saude Publica. 2012 Sep;28(9):1772-84.

10. Menegazzo IR, Pereira MR, Villalba JP. Levantamento epidemiológico de doenças relacionadas à fisioterapia em uma Unidade Básica de Saúde do município de Campinas. J Health Sci Inst. 2010 outdez;28(4):348-51.

11. Corti CM, Rigon C. Epidemiology of osteoarthritis: prevalence, risk factors and functional impact. Aging Clin Exp Res. 2003 Oct;15(5):359-63.

12. Martinez JE, Santos, BZC, Fasolin RP, Gianini RJ. Perfil de pacientes com queixa de dor músculo-esquelética em unidade básica em Sorocaba. Rev Soc Bras Clin Med. 2008;6(5):167-71.

13. Henschke N, Maher CG, Refshauge KM, Herbert RD, Cumming RG, Bleasel J, et al. Prognosis in patients with recent onset low back pain in Australian primary care: inception cohort study. BMJ. 2008 Jul;337(171):1-7.
14. Wofford JL, Mansfield RJ, Watkins RS. Patient characteristics and clinical management of patients with shoulder pain in U.S. primary care settings: secondary data analysis of the National Ambulatory Medical Care Survey. BMC Musculoskelet Disord. 2005 Feb;6(4):1-6.

15. Baena CP, Soares MCF. Subsídios reunidos junto à equipe de saúde para a inserção da fisioterapia na Estratégia Saúde da Família. Fisioter Mov. 2012 abrjun;25(2):419-31.

16. Bispo Júnior JP. Physiotherapy and collective health: challenges and new professional responsibilities. Cien Saude Coletiva. 2010 Jun;15 Suppl 1:1627-36.

17. Cott CA, Mandoda S, Landry MD. Models of integrating physical therapists into family health teams in Ontario, Canada: challenges and opportunities. Physiother Can. 2011 Aug;63(3):265-75.

Recebido em 18/07/2014

Aprovado em 14/01/2015 